\title{
Kinetic Studies on Drug Disposition in Rabbits. I. Renal Excretion of Iodopyr- acet and Sulfamethizole
}

\author{
Kazunori KATAYAMA, Hiroyuki OHTANI, Toshihiko KAWABE, Hiromoto MIZUNO, \\ Makiko ENDOH, Masawo KAKEMI, and Tamotsu KOIZUMI
}

Faculty of Pharmaceutical Sciences, Toyama Medical and Pharmaceutical University, Sugitani 2630, Toyama, 930-01, Japan

(Received July 21, 1989)

In order to quantify the renal handling of iodopyracet (IOD) and sulfamethizole (SMZ), singledrug clearance studies in rabbits were performed under quasi-steady state conditions with stepwise increasing the infusion rate of IOD or SMZ. Although concentration dependence of plasma protein binding was observed for both drugs, the urinary excretion rate of IOD was proportional to its total plasma concentration at low total plasma concentrations of $0.05-0.8 \mathrm{mM}$. On the other hand, the relationship between urinary excretion rate and total plasma concentration of SMZ was a concave-ascending curve at low plasma concentrations and the renal clearance of $\mathrm{SMZ}$ was sensitive to changes in plasma protein binding. However, renal clearances referenced to unbound plasma concentration at total plasma concentrations of $0.05 \mathrm{mM}$ for IOD and SMZ were 9.5 and $38 \mathrm{l} / \mathrm{h}$, respectively. Those values were much greater than the effective plasma flow in rabbits. These facts indicated that the intrinsic clearances at the sites of tubular secretion were high and that the rates of secretion were fully or partially limited by the renal plasma flow. Furthermore it was suggested that unbound drug was liberated from plasma protein at the sites of tubular secretion. The data obtained at high plasma concentrations indicated that the tubular secretion of IOD had capacity limited characteristics and that the urinary excretion of SMZ involved tubular reabsorption as well as saturable tubular secretion. From the data obtained, a perfusion-limited pharmacokinetic model was constructed characterizing the excretory processes, namely, glomerular filtration, passive tubular reabsorption, saturable tubular secretion and reequilibrium between bound and unbound drugs in plasma. For both drugs, the estimates for bulk flow rate were reasonable values of effective renal plasma flow and the dissociation constants for tubular secretion agreed well with those for in vitro renal cortex accumulation, suggesting that the kinetic model based on physiological concepts was useful for the understanding of the drug elimination processes.

Keywords - iodopyracet; sulfamethizole; renal transport; tubular secretion; renal cortex slice; protein binding; perfusion limited model

\section{Introduction}

Renal excretion involves three transport processes in relation to the renal anatomy, i.e., glomerular filtration, renal tubular secretion, and tubular reabsorption. These transport processes are closely related to each other and determine drug and metabolite levels in the body. However the systematic and kinetic studies incorporating the functional characteristics of these processes have been limited, ${ }^{1-7)}$ because the determination of drug concentrations at the sites of the transport mechanisms in the inner part of kidney is very difficult experimentally.

Perfusion-limited models based on physiology, anatomy, and biochemistry have been developed recently. ${ }^{8-10)}$ The roles of blood flow, the intrinsic ability of the organ to remove drug, and binding within blood, all of which influence drug elimination, have been determined. These approaches have been applied to analyze quantitatively the renal excretion of some drugs, which were excreted by means of glomerular filtration and tubular secretion. ${ }^{4,6)}$ On the other hand, for drugs involving glomerular filtration and tubular reabsorption, the relationship between the magnitude of reabsorption and the physicochemical properties of drug, or the dependence of renal clearance on urine flow rate have been investigated using different models based on physiological considerations. ${ }^{11-13)}$

However, many drugs have being excreted by means of both tubular secretion and tubular reabsorption. Therefore, to develop a more comprehensive model which can be applied to drugs excreted by means of not only glomerular 
filtration and secretion but also tubular reabsorption is desirable. The purpose of this investigation was to present the quantitative relationship between plasma drug concentration and renal excretion using a perfusion-limited model. This model involved the three excretory processes in renal handling of drugs in rabbits. For this purpose, as a model drug, we used sulfamethizole (SMZ), which is known to be excreted by glomerular filtration, saturable tubular secretion and passive tubular reabsorption. ${ }^{13,14)}$ Furthermore, we examined also the renal excretion of iodopyracet (IOD) involving glomerular filtration and tubular secretion. ${ }^{4)}$

\section{Theoretical}

To develop a kinetic model of the renal excretion at quasi-steady state which is able to account for various aspects of renal transport mechanisms, the following assumptions have been made: 1) The kidney is composed of three compartments, i.e., glomerulus, capillary vessel, and tubular lumen compartments, 2) the capillary and lumen compartments are well-stirred so that the concentrations of drug in capillary and lumen are equal to the concentrations in the emergent body fluids, i.e., those of the renal venous plasma and the urine, respectively, 3 ) the dissociation of bound drug from the binding protein in plasma is faster than the renal vascular passage, 4) tubular reabsorption involves passive diffusion. When the difference in $\mathrm{pH}$ values between the capillary vessel and tubular lumen is negligible, the rate of reabsorption is proportional to the difference between the concentration of unbound drug in the capillary compartment and the concentration of drug in the tubular lumen compartment, 5) tubular secretion is nonlinear carrier-mediated transport and the rate of secretion obeys the Michaelis-Menten equation with respect to the concentration of unbound drug in the capillary compartment, 6) reabsorption of water takes place in the more proximal region than the site of secretion and reabsorption of drug.

The model for the renal excretion of drug is depicted in Fig. 1.

Based on assumptions described above, the mass balance equation for the drug in the capillary compartment is given as follows:

$$
\begin{aligned}
& {\left[Q_{\mathrm{p}} C_{\mathrm{p}, \text { in }}-\mathrm{GFR} f_{\mathrm{p}, \text { in }} C_{\mathrm{p}, \text { in }}\right]-\left(Q_{\mathrm{p}}-\mathrm{UR}\right) C_{\mathrm{p}, \text { out }}} \\
& =\frac{V_{\mathrm{m}} f_{\mathrm{p}, \text { out }} C_{\mathrm{p}, \text { out }}}{K_{\mathrm{m}}+f_{\mathrm{p}, \text { out }} C_{\mathrm{p}, \text { out }}}+\mathrm{CL}_{\mathrm{re}}\left(f_{\mathrm{p}, \text { out }} C_{\mathrm{p}, \text { out }}-C_{\mathrm{u}}\right)
\end{aligned}
$$

where $C_{\mathrm{p} \text {,in }}, C_{\mathrm{p} \text {,out }}$ and $C_{\mathrm{u}}$ are the total drug concentrations in the artery, the vein and the urine; $Q_{\mathrm{p}}, \mathrm{GFR}$ and UR are the renal plasma flow, the glomerular filtration rate and the urine flow; $f_{\mathrm{p} \text {,in }}$ and $f_{\mathrm{p} \text {,out }}$ are the unbound fractions of drug in the artery and the vein; $V_{\mathrm{m}}$ is the maximal transport capacity of secretion; $K_{\mathrm{m}}$ is the Michaelis-Menten constant of the drug-carrier complex and $\mathrm{CL}_{\mathrm{re}}$ is the proportionality constant of passive reabsorption (in units of $1 / h$ ). The terms in the brackes and the last term of the left

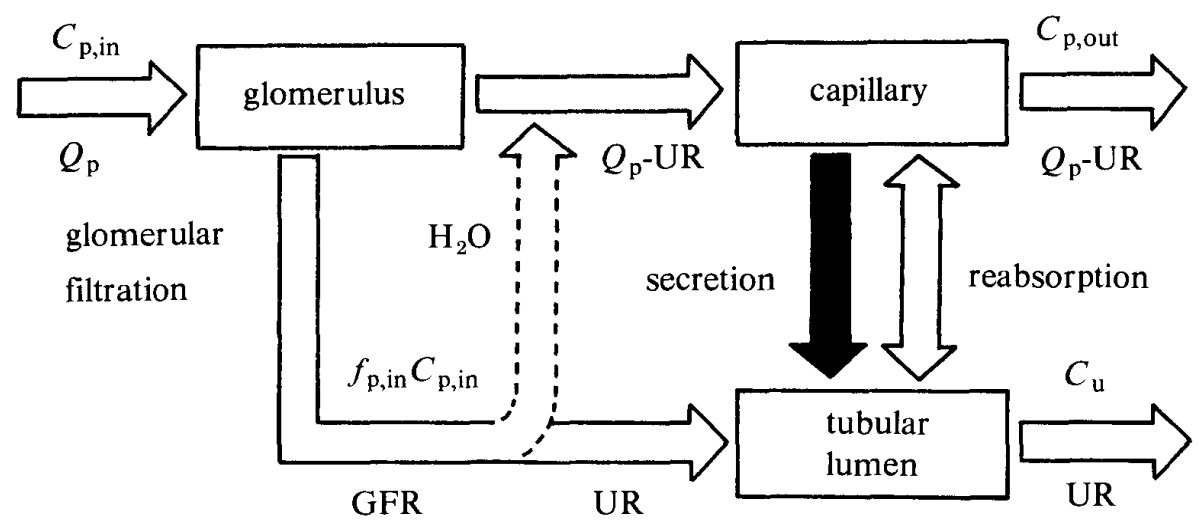

Fig. 1. Schematic Representation of Model of Renal Excretion

The arrow indicates the flow of drug (s) or water and the meanings of the parameters are shown in the text. 
side of Eq. 1 represent the rate of inflow at which drug enters the capillary compartment and the rate of outflow at which drug leaves the capillary compartment through the renal vein, respectively.

The urinary excretion rate $\left(d X_{\mathrm{u}} / d t\right)$ at steady state is given as follows:

$$
\frac{d X_{\mathrm{u}}}{d t}=Q_{\mathrm{p}} C_{\mathrm{p}, \text { in }}-\left(Q_{\mathrm{p}}-\mathrm{UR}\right) C_{\mathrm{p}, \text { out }}
$$

so that

$$
\begin{aligned}
\frac{d X_{\mathrm{u}}}{d t}= & \operatorname{GFR} f_{\mathrm{p}, \text { in }} C_{\mathrm{p}, \text { in }}+\frac{V_{\mathrm{m}} f_{\mathrm{p}, \text { out }} C_{\mathrm{p}, \text { out }}}{K_{\mathrm{m}}+f_{\mathrm{p}, \text { out }} C_{\mathrm{p}, \text { out }}} \\
& +\mathrm{CL}_{\mathrm{re}}\left(f_{\mathrm{p}, \text { out }} C_{\mathrm{p}, \text { out }}-C_{\mathrm{u}}\right)
\end{aligned}
$$

Since the urinary excretion rate equals the product of the urine flow and the urinary drug concentration, substituting $\left(d X_{\mathrm{u}} / d t\right) / \mathrm{UR}$ for $C_{\mathrm{u}}$ and appropriate rearrangement gives

$$
\begin{aligned}
\frac{d X_{\mathrm{u}}}{d t} & =\left(\mathrm{GFR} f_{\mathrm{p}, \text { in }} C_{\mathrm{p}, \text { in }}+\frac{V_{\mathrm{m}} f_{\mathrm{p}, \text { out }} C_{\mathrm{p}, \text { out }}}{K_{\mathrm{m}}+f_{\mathrm{p}, \text { out }} C_{\mathrm{p}, \text { out }}}\right. \\
& \left.+\mathrm{CL}_{\mathrm{re}} f_{\mathrm{p}, \text { out }} C_{\mathrm{p}, \text { out }}\right)\left(1-\frac{\mathrm{CL}_{\mathrm{re}}}{\mathrm{UR}+\mathrm{CL}_{\mathrm{re}}}\right)(4)
\end{aligned}
$$

Otherwise, renal clearance $\left(\mathrm{CL}_{R}\right)$ is defined as follows:

$$
\begin{aligned}
\mathrm{CL}_{\mathrm{R}} & =\frac{d X_{\mathrm{u}} / d t}{C_{\mathrm{p}, \text { in }}} \\
& =\frac{Q_{\mathrm{p}} C_{\mathrm{p}, \text { in }}-\left(Q_{\mathrm{p}}-\mathrm{UR}\right) C_{\mathrm{p}, \text { out }}}{C_{\mathrm{p}, \text { in }}} \\
& =Q_{\mathrm{p}}-\left(Q_{\mathrm{p}}-\mathrm{UR}\right)\left(\frac{C_{\mathrm{p}, \text { out }}}{C_{\mathrm{p}, \text { in }}}\right)
\end{aligned}
$$

which can be written, according to Eq. 4, by

$$
\begin{aligned}
\mathrm{CL}_{\mathrm{R}}= & {\left[\mathrm{GFR} f_{\mathrm{p}, \text { in }}+f_{\mathrm{p}, \text { out }} Z\left(\frac{C_{\mathrm{p}, \text { out }}}{C_{\mathrm{p}, \text { in }}}\right)\right] . } \\
& {\left[1-\frac{\mathrm{CL}_{\mathrm{re}}}{\mathrm{UR}+\mathrm{CL}_{\mathrm{re}}}\right] }
\end{aligned}
$$

in which
$Z=\frac{V_{\mathrm{m}}}{K_{\mathrm{m}}+f_{\mathrm{p}, \text { out }} C_{\mathrm{p}, \text { out }}}+\mathrm{CL}_{\mathrm{re}}$

Substituting Eq. 6 into 5 and eliminating the concentration ratio terms and appropriate rearrangement yields

$$
\begin{aligned}
\mathrm{CL}_{\mathrm{R}}= & {\left[1-\frac{\mathrm{CL}_{\mathrm{re}}}{\mathrm{UR}+\mathrm{CL}_{\mathrm{re}}}\right] . } \\
& {\left[\frac{\left(Q_{\mathrm{p}}-\mathrm{UR}\right) \mathrm{GFR} f_{\mathrm{p}, \text { in }}+Q_{\mathrm{p}} f_{\mathrm{p}, \text { out }} Z}{\left(Q_{\mathrm{p}}-\mathrm{UR}\right)+f_{\mathrm{p}, \text { out }} Z 1-\left(\frac{\mathrm{CL}_{\mathrm{re}}}{\mathrm{UR}+\mathrm{CL}_{\mathrm{re}}}\right)}\right] }
\end{aligned}
$$

In Eq. 7, the unbound fractions $\left(f_{\mathrm{p}, \text { in }}\right.$ and $\left.f_{\mathrm{p}, \text { out }}\right)$ and the drug concentrations in the effluent $\left(C_{\mathrm{p}, \text { out }}\right)$ are dependent upon the total concentration of drug in the influent. Details of the estimation procedure of these values are presented in Appendix.

An overall drug disposition model is depicted in Fig. 2. It is postulated that clearance process for extra-renal elimination is dependent only on the unbound drug concentration. The differential equation describing the rate of change in the amount of drugs in the body is given by

$$
\frac{d X_{\mathrm{b}}}{d t}=R_{0}-\mathrm{CL}_{\mathrm{R}} C_{\mathrm{p}, \text { in }}-\frac{V_{\mathrm{met}} f_{\mathrm{p}, \text { in }} C_{\mathrm{p}, \text { in }}}{K_{\mathrm{met}}+f_{\mathrm{p}, \text { in }} C_{\mathrm{p}, \text { in }}}
$$

where $X_{\mathrm{b}}$ is the amount of drug in the body and $R_{0}$ is infusion rate, and $V_{\text {met }}$ and $K_{\text {met }}$ are the maximal capacity for extra-renal clearance and the corresponding dissociation constant, respectively. The plasma concentration of IOD is expressed with the volume of distribution. $\left(V_{\mathrm{d}}\right)$ by

$$
C_{\mathrm{p}, \text { in }}=X_{\mathrm{b}} / V_{\mathrm{d}}
$$

For SMZ, drug movement within the body was kinetically heterogeneous and the change of the total amount of drug with time is expressed by the relationship,

$$
\frac{d X_{\mathrm{b}}}{d t}=\frac{d(\text { blood pool })}{d t}+\frac{d(\mathrm{TRE})}{d t}+\frac{d(\mathrm{TSE})}{d t}
$$




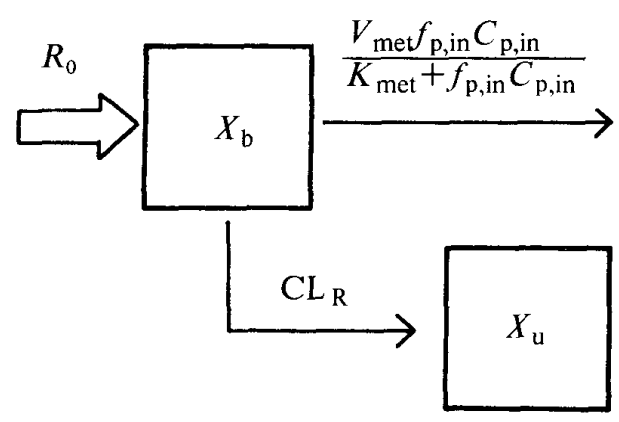

Fig. 2. Diagrammatic Representation of a Pharmacokinetic Model of IOD or SMZ Disposition in Rabbits

It is assumed the rates of non-renal elimination obeys the Michaelis-Menten equation with respect to the concentration of unbound drug and renal excretion occurs as the perfusion limited fashions as shown in the text.

where TRE and TSE are tissue rapidly and slowly equilibrated with the blood pool, respectively. The description and parameters for drug movements within the body were adopted from the subsequent paper. ${ }^{15)}$ The plasma concentration of SMZ, therefore, is given using the whole blood concentration $\left(C_{\mathrm{b}}\right)$ and red blood cell concentration $\left(C_{\text {red }}\right.$ ) in the blood pool as follows:

$$
C_{\mathrm{p}, \text { in }}=\left(C_{\mathrm{b}}-\mathrm{Hct} C_{\mathrm{red}}\right) /(1-\mathrm{Hct})
$$

where Hct is the hematocrit.

\section{Materials and Method}

Materials - SMZ (Eisai Co., Ltd., Tokyo, Japan), IOD (3,5-diiodo-4-pyridone-1-acetic acid: Aldrich Chemical Co., Milwaukee, Wis.) and tolbutamide (TB: Ono Pharmaceutical Co., Osaka) were obtained commercially. All other chemicals were of reagent grade and used without further purification.

$$
\begin{aligned}
& C_{\mathrm{p}}^{\mathrm{A}}=C_{\mathrm{f}}^{\mathrm{A}}+ \\
& \frac{N_{1}(\mathrm{p}) C_{\mathrm{f}}^{\mathrm{A}}}{K_{1}^{\mathrm{A}}\left(1+C_{\mathrm{f}}^{\mathrm{B}} / K_{1}^{\mathrm{B}}\right)+C_{\mathrm{f}}^{\mathrm{A}}}+\frac{N_{2}(\mathrm{p}){ }^{\mathrm{A}} C_{\mathrm{f}}^{\mathrm{A}}}{K_{2} \mathrm{~A}+C_{\mathrm{f}}^{\mathrm{A}}}
\end{aligned}
$$

Renal Cortex Accumulation in Vitro Rabbits anesthetized with urethane were exsanguinated and their kidneys were perfused with ice-cold physiological saline solution. Renal cortex slices, approximately $0.5 \mathrm{~mm}$ thick, were prepared from kidneys by means of a Stadie-
Riggs hand slicer and kept in ice-cold saline bubbled with a mixture of $95 \% \mathrm{O}_{2}$ and $5 \% \mathrm{CO}_{2}$ until use. After a brief blotting on filter paper, the slices, approximately $300 \mathrm{mg}$, were added to 2.7 $\mathrm{ml}$ of a suspending medium. The suspending médium had the following composition: $\mathrm{NaCl}$ $88.7 \mathrm{mM}, \mathrm{KCl} 40 \mathrm{mM}$, sodium phosphate buffer (pH 7.4) $7.4 \mathrm{mM}$, sodium acetate $11.1 \mathrm{mM}$ and $\mathrm{CaCl}_{2} \quad 0.74 \mathrm{mM}$. The final osmotic pressure of the medium containing drug was adjusted to 283 $\mathrm{mOsm} / 1$ by adding $\mathrm{NaCl}$. The initial concentrations of SMZ or IOD in the medium were $0.0148-1.48 \mathrm{mM}$. The beakers were shaken at $25^{\circ} \mathrm{C}$ and 100 oscillations per min for $2 \mathrm{~h}$ with the gas space displaced with mixed gas of $95 \%$ $\mathrm{O}_{2}$ and $5 \% \mathrm{CO}_{2}$, or $100 \% \mathrm{~N}_{2}$ gas. At the end of the incubation period, the beakers were moved into cracked-ice and the slices were promptly removed from the medium, blotted on filter paper and weighed. For SMZ, after the addition of 5 $\mathrm{ml}$ of $10 \%$ trichloroacetic acid (TCA), the slices were crushed with a spatula and shaken for 30 min and centrifuged. For precipitation of the protein in the medium, an equal quantity of $10 \%$ TCA was added to the medium solution. For the assay of IOD, the slices were crushed and shaken after the addition of $5 \mathrm{ml}$ of ethyl acetate as described above.

Plasma Protein Binding - Ultrafiltration of the plasma samples from rabbits following administration of drugs was performed in membrane cones (Centriflo model CF50A, Amicon Corp., Danvers, Mass). For the characteristic of plasma protein binding, it is assumed that IOD, SMZ and TB have common high affinity sites and two drugs bind competitively to these sites. Therefore, in a case of two drugs, A and B, the binding isotherms are as follows:

$$
\begin{aligned}
& C_{\mathrm{p}}^{\mathrm{B}}=C_{\mathrm{f}}^{\mathrm{B}}+ \\
& \frac{N_{1}(\mathrm{p}) C_{\mathrm{f}}^{\mathrm{B}}}{K_{1}{ }^{\mathrm{B}}\left(1+C_{\mathrm{f}}^{\mathrm{A}} / K_{1}{ }^{\mathrm{A}}\right)+C_{\mathrm{f}}^{\mathrm{B}}}+\frac{N_{2}(\mathrm{p})^{\mathrm{B}} C_{\mathrm{f}}^{\mathrm{B}}}{K_{2}{ }^{\mathrm{B}}+C_{\mathrm{f}}^{\mathrm{B}}}
\end{aligned}
$$

where $C_{\mathrm{p}}$ and $C_{\mathrm{f}}$ are the total and unbound drug concentrations, $N_{1}(\mathrm{p})$ and $N_{2}(\mathrm{p})$ are the capacities of binding sites, and $K_{1}$ and $K_{2}$ are the dissociation constants for these sites. Superscripts (A and B) mean drug A and B, respectively. For the estimation of the binding parameters, Eqs. 
12,13 were simultaneously fitted to the five sets of data, i.e., IOD alone, SMZ alone, TB alone, the combination of IOD and SMZ, and the combination of SMZ and TB.

Clearance Studies - Unanesthetized male albino rabbits weighing 3.0 to $4.0 \mathrm{~kg}$ were used. The animals were fixed on the supine position during the experiment. Physiological saline solution (containing $0.2 \mathrm{~g}$ of inulin per $100 \mathrm{ml}$ ) was infused through the right marginal ear vein at a rate of $100 \mathrm{ml} / \mathrm{h}$ to insure an adequate hydration and urine flow $(80-110 \mathrm{ml} / \mathrm{h})$ and to measure GFR. The measurement of the renal clearance of SMZ or IOD was made at different plasma levels, i.e., eight infusion rates (SMZ: 25, 100, $200,300,600,1000,1500$, and $2000 \mathrm{mg} / \mathrm{h}$, IOD: $100,200,300,600,1000,1500,2000$, and $2500 \mathrm{mg} / \mathrm{h}$ ). These drugs were given in ascending order at a constant flow rate $(100 \mathrm{ml} / \mathrm{h})$ and the infusion interval of each drug solution was 1.0 or $1.5 \mathrm{~h}$. Urine samples were collected with Nelaton's catheter, which was introduced into the bladder. The sampling interval was $30 \mathrm{~min}$ and, accounting for the lag in the appearance of drug in the urine, each sampling period was delayed for $1 \mathrm{~min}^{14)}$ from the time-scale of drug infusion intervals. A blood sample was drawn through a heparinized syringe from the left marginal ear vein at $1 \mathrm{~min}$ before the midpoint of each urine collection interval.

Analytical Method - The concentration of IOD was determined by a modification of the method of Hekman et al. ${ }^{16)} 0.2 \mathrm{ml}$ of sample was added to $1 \mathrm{ml}$ of $\mathrm{H}_{2} \mathrm{O}, 0.5 \mathrm{ml}$ of $1 \mathrm{~N} \mathrm{HCl}$ and $0.1 \mathrm{ml}$ of $200 \mu \mathrm{g} / \mathrm{ml}$ hippuric acid (as internal standard). After extraction with $5 \mathrm{ml}$ of ethyl acetate, $4 \mathrm{ml}$ of the organic layer was pipetted into another tube and evaporated to dryness. The residue was reconstituted in $0.5 \mathrm{ml}$ of methanol and $5 \mu \mathrm{l}$ was injected into the column. A Shimadzu Model LC-6A high performance liquid chromatograph system equipped with a SPD-6A absorbance detector (Shimadzu, Kyoto, Japan) and LiChrosorb RP-18 (15 cm $\times$ $4 \mathrm{~mm}$ i.d., $5 \mu \mathrm{m}$ particle size; Merck, Darmstadt, West Germany) was used. The mobile phase was water (containing $10 \mathrm{mM}$ tetrabutylammonium sulfate and $10 \mathrm{~mm}$ Tris) and methanol $(70: 30, \mathrm{v} / \mathrm{v})$. The flow rate was $0.8 \mathrm{ml} / \mathrm{min}$, the oven temperature was $40^{\circ} \mathrm{C}$, and the wavelength of the detector was $238 \mathrm{~nm}$.

TB was determined by a modification of the method of Wahlin et al. ${ }^{17)} 0.1 \mathrm{ml}$ of sample was added to $0.2 \mathrm{ml}$ of $\mathrm{H}_{2} \mathrm{O}, 0.2 \mathrm{ml}$ of $1 \mathrm{~N} \mathrm{HCl}, 0.1$ $\mathrm{ml}$ of $100 \mu \mathrm{g} / \mathrm{ml}$ chlorpropamide (as internal standard) and $5 \mathrm{ml}$ of dichloromethane. After extraction and centrifugation, the organic layer was evaporated to dryness. The residue was redissolved in methanol and injected into the column. The column was Lichrosorb Rp-18 (5 $\mu \mathrm{m}, 15 \mathrm{~cm} \times 4.0 \mathrm{~mm}$ i.d.). The mobile phase was $0.01 \mathrm{M}$ phosphate buffer (pH 3.5): methanol (48:52) and the flow rate was $1.0 \mathrm{ml} / \mathrm{min}$. The chromatography waw carried out at ambient temperature and the wavelength of the ultraviolet (UV) detector was set at $230 \mathrm{~nm}$.

SMZ concentrations were determined colorimetrically in the same manner as given in the previous report. ${ }^{18)}$ Inulin concentrations in plasma and urine were estimated colorimetrically according to the method of Dische et al. ${ }^{19}$ )

Data Analysis - In renal clearance studies, experimental data were fitted to Eqs. 4, 8-11 and 1A (see Appendix) using a nonlinear least square regression program based on the algorithm of Berman et $a l^{20}$ ) Weighting factors were inversely proportional to the variances of plasma concentration at each time and the amount of drug excreted in urine in each sampling interval. For SMZ, the disposition parameters other than renal and extra-renal elimination, were adopted from the subsequent paper in regard of the tissue distribution of SMZ. ${ }^{15}$

\section{Results}

\section{In Vitro Renal Slice Accumulation of IOD and SMZ}

The uptake of SMZ or IOD by renal cortex slices was studied under aerobic conditions. The results were expressed in terms of the concentrations in slices against the concentrations in the medium after $2 \mathrm{~h}$ of incubation (Fig. 3). It was indicated that the overall uptake by the cortex of each drug was constituted of a single Langmuir's saturable uptake and a non-saturable uptake, i.e., 

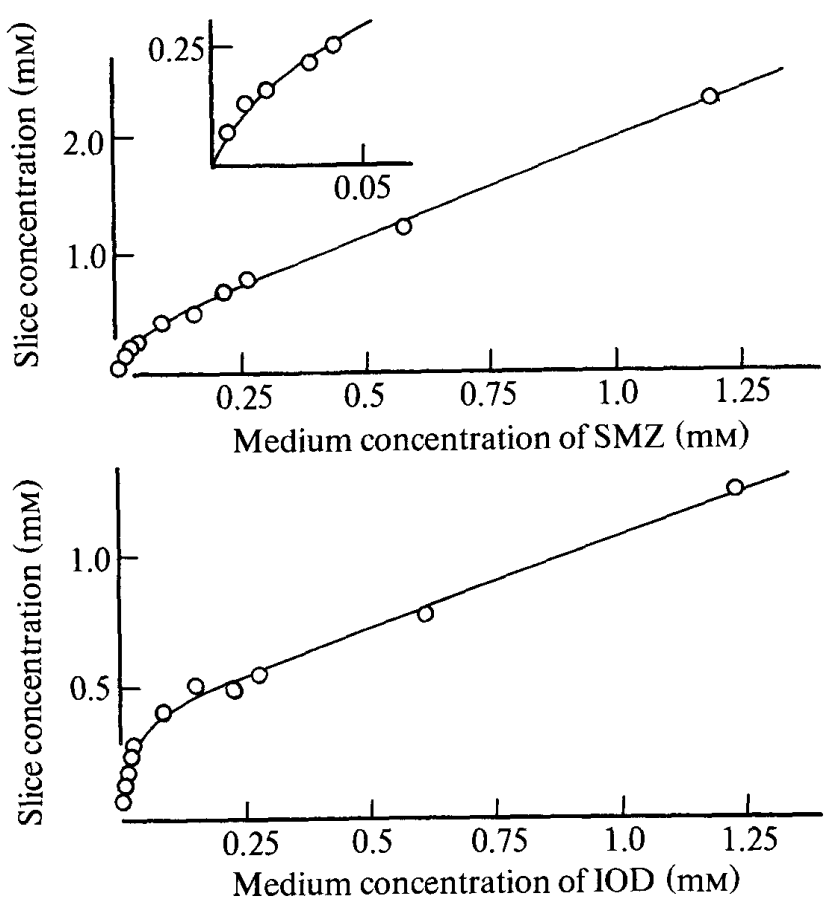

Fig. 3. Concentration Dependence of SMZ and IOD Uptakes by Renal Cortex Slices under Aerobic Conditions

Each point represents the mean of two determinations after $2 \mathrm{~h}$ of incubation and solid lines represent the calculated values with nonlinear regression analysis using Eq. 14 with a weighting factor of the reciprocal of data. Upper panel: SMZ (the inset shows the results under lower medium concentration). Lower panel: IOD.

$$
C_{\mathrm{S}}=\frac{B_{\mathrm{T}} C_{\mathrm{M}}}{K_{\mathrm{d}}+C_{\mathrm{M}}}+f_{\mathrm{B}} C_{\mathrm{M}}
$$

where $C_{\mathrm{S}}$ and $C_{\mathrm{M}}$ are the final concentrations in the slice and medium, $B_{\mathrm{T}}$ and $K_{\mathrm{d}}$ are the maximum capacity and the dissociation constant for the saturable uptake and $f_{\mathrm{B}}$ is the proportionality constant for the linear uptake. The parameters of both drugs estimated form nonlinear regression analysis using Eq. 14 were shown in Table I and the simulated values were also shown in

TABLE I. Estimated Parameter Values of IOD and SMZ Uptakes by Renal Cortex Slices ${ }^{a}$ )

\begin{tabular}{lcc}
\hline & IOD & SMZ \\
\hline$B_{\mathrm{T}}(\mu \mathrm{M})$ & $406.0 \pm 25.0$ & $344.0 \pm 41.0$ \\
$K_{\mathrm{d}}(\mu \mathrm{M})$ & $18.5 \pm 3.8$ & $31.1 \pm 8.6$ \\
$f_{\mathrm{B}}$ & $0.672 \pm 0.034$ & $1.63 \pm 0.07$ \\
\hline
\end{tabular}

a) Values are presented as mean \pm S.D.

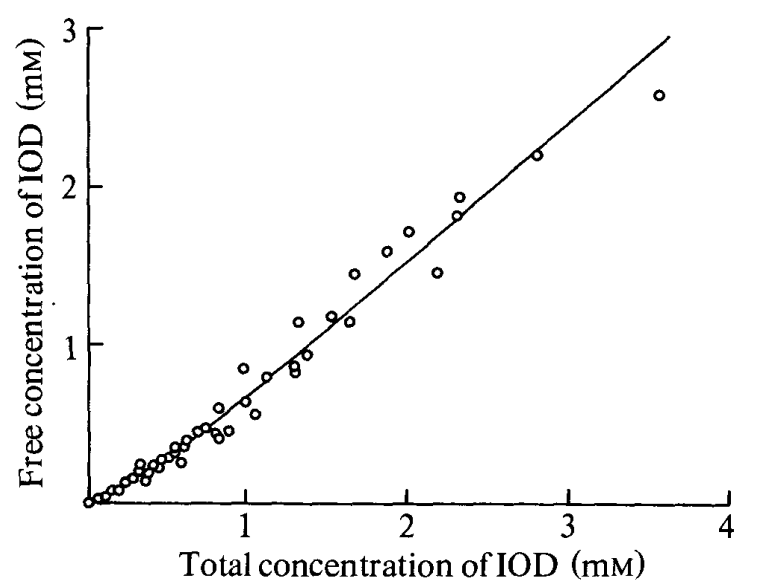

Fig. 4. Relationship between Unbound Plasma Concentrations and Total Plasma Concentrations of IOD

The solid line is the simulated value using the binding parameters in Table II.

Fig. 3, as solid lines. Although the linear uptake of SMZ was higher than that of IOD, the dissociation constants of SMZ and IOD were 31 and 19 $\mu \mathrm{M}$, respectively, suggesting that, in regard of the saturable binding to renal slices, IOD has a higher affinity than SMZ.

Under anaerobic conditions, the saturable bindings of SMZ and IOD were abolished completely and the overall uptake could not be clearly distinguished from the linear uptake (data not shown). These facts suggested that the linear uptake reflected non-specific physical binding to renal constituents and the Langmuir's saturable

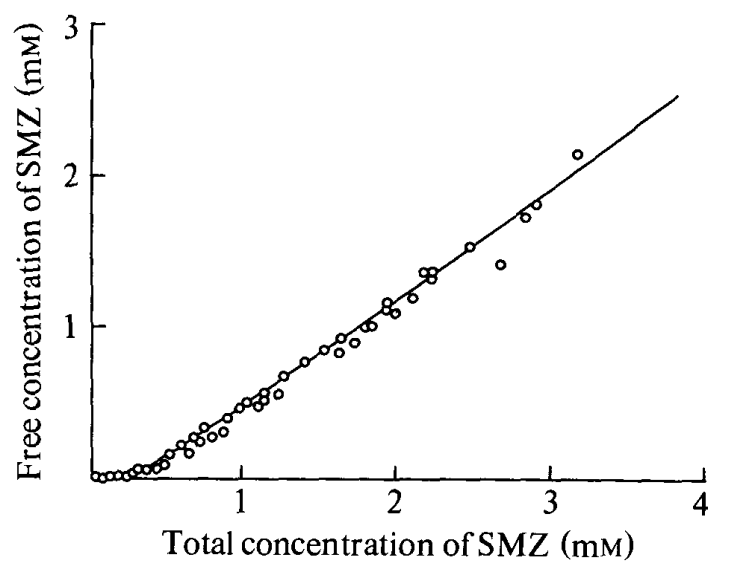

Fig. 5. Relationship between Unbound Plasma Concentrations and Total Plasma Concentrations of SMZ

The solid line is the simulated value using the binding parameters in Table II. 
uptake was energy dependent active accumulation.

\section{Plasma Protein Binding of IOD, SMZ and TB}

The in vivo plasma protein binding of IOD, SMZ or TB was determined by the ultrafiltration method. Figure 4 shows the plasma protein binding of IOD. The unbound fractions of IOD were about $0.37,0.65$, and 0.77 at the total plasma concentrations of $0.05,0.8$ and $2.0 \mathrm{mM}$, respectively, and concentration dependence of plasma protein binding was observed.

The plasma protein binding of SMZ is shown in Fig. 5. Pronounced concentration dependence of plasma protein binding was observed and the unbound fractions of SMZ were about 0.04 , 0.43 and 0.59 at the total plasma concentrations of $0.05,0.8$ and $2.0 \mathrm{mM}$, respectively.

The nonlinear plasma protein binding of TB was also observed as shown in Fig. 6. The parameters of three drugs estimated from nonlinear regression analysis using Eqs. 12, 13 are shown in Table II and the simulated values for the binding study of a single drug are also shown in Figs. 4-6, as solid lines. The validity of the estimated parameters for the drug-drug interaction studies will be discussed in subsequent papers. ${ }^{21,22)}$

\section{In Vivo Clearance Studies}

In order to obtain the plasma and urinary excretion data under quasi-steady state conditions, in vivo clearance studies of IOD were performed by changing the infusion rate in steps, given in ascending order from 100 to $2500 \mathrm{mg} / \mathrm{h}$. Plasma concentration and urinary excretion rate profiles

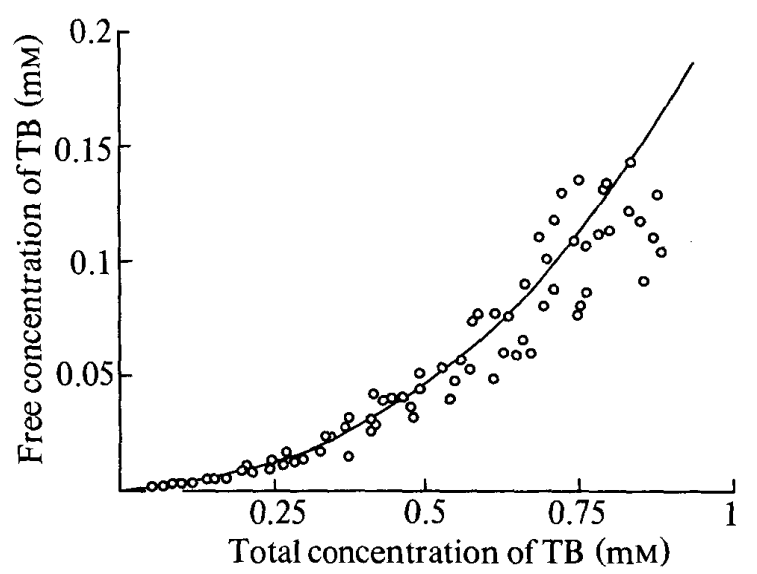

Fig. 6. Relationship between Unbound Plasma Concentrations and Total Plasma Concentrations of TB

The solid line is the simulated value using the binding parameters in Table II.

of IOD are shown in Fig. 7. The solid lines are the calculated values obtained by the nonlinear regression analysis.

Figure 8 shows the plasma concentrations and urinary excretion rates of SMZ during eight different infusion rates from 25 to $2000 \mathrm{mg} / \mathrm{h}$ and the calculated values are also shown as solid lines.

The estimated parameters obtained with data of IOD and SMZ are shown in Table III. In the listed parameters, GFR and UR were experimentally determined. The parameter value $\left(\mathrm{CL}_{\mathrm{re}}\right)$ on the renal tubular reabsorption of IOD was fixed to zero, because no data supporting reabsorption of IOD was available.

TABLE II. Estimated Parameter Values of Plasma Protein Binding of IOD, SMZ and TB ${ }^{a}$ )

\begin{tabular}{lcccc}
\hline Drug & $\begin{array}{c}N_{1}(\mathrm{p})^{b)} \\
(\mathrm{mM})\end{array}$ & $\begin{array}{c}K_{1} \\
(\mathrm{mM})\end{array}$ & $\begin{array}{c}N_{2}(\mathrm{p}) \\
(\mathrm{mM})\end{array}$ & $\begin{array}{c}K_{2} \\
(\mathrm{mM})\end{array}$ \\
\hline IOD & $0.306 \pm 0.022$ & $0.179 \pm 0.034$ & $1116 \pm 12116$ & $8870 \pm 96340$ \\
SMZ & $0.306 \pm 0.022$ & $0.0106 \pm 0.0026$ & $4.10 \pm 2.93$ & $8.40 \pm 7.13$ \\
TB & $0.306 \pm 0.022$ & $0.00893 \pm 0.00210$ & $0.814 \pm 0.132$ & $0.142 \pm 0.046$ \\
\hline
\end{tabular}

a) Values are presented as mean \pm S.D. b) It was assumed that the parameter was the identical value for IOD, SMZ and TB, and two drugs bound competitively to these affinity sites. The coefficient of determination of the estimated and calculated total concentration in plasma (with the range and mean of observed total concentrations (mM) in brackets) was as follows: SMZ alone $(n=90), r^{2}=0.994(0.023-3.15,0.785)$ : IOD alone $(n=84), r^{2}=0.987(0.0057-4.11,0.686):$ TB alone $(n=$ $80), r^{2}=0.962(0.0526-0.885,0.488):$ combination of SMZ and IOD $(n=41), r^{2}=0.701(0.189-0.541 ; 0.341)$ for SMZ; $r^{2}=0.991(0.113-4.62,1.30)$ for IOD: combination of SMZ and TB $(n=78), r^{2}=0.987(0.035-2.61,0.426)$ for SMZ; $r^{2}=0.710(0.298-0.989,0.622)$ for TB. 


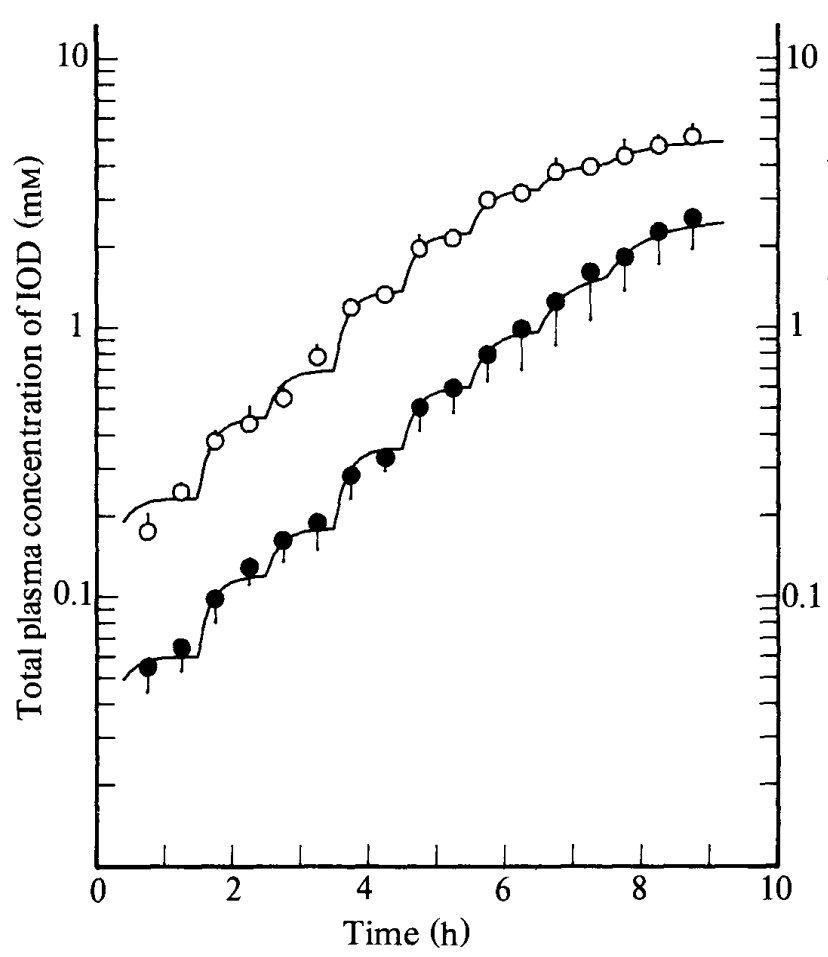

Fig. 7. Time Course of Plasma Concentration and Urinary Excretion Rate of IOD during Changing the Infusion Rate in Steps

The plotted points represent the observed data (open circle, urinary excretion rate; closed circle, total plasma concentration) and the solid lines represent calculated values. Each experimental point is shown as the mean \pm S.D. $(n=4)$.

\section{Discussion}

Perfusion-limited models have recently been applied to the description of the renal excretion of a drug with tubular secretion, ${ }^{3,4,6,23)}$ as the clearance of an eliminating organ is determined by the perfusion rate and the intrinsic ability of the organ to remove drug. In the present study, the perfusion-limited model was constructed to quantify the renal handling of SMZ, which was excreted by means of glomerular filtration, saturable tubular secretion and passive tubular reabsorption.

As shown in Table III, the estimated bulk flow $\left(Q_{\mathrm{p}}\right)$ of SMZ as well as IOD gave reasonable values with respect to the renal effective plasma flow in the rabbit. For IOD, this is in agreement with the study in the dog. ${ }^{4)} \mathrm{SMZ}$ was incorporated into the erythrocytes and the ratio of the erythrocytes to the unbound plasma con-

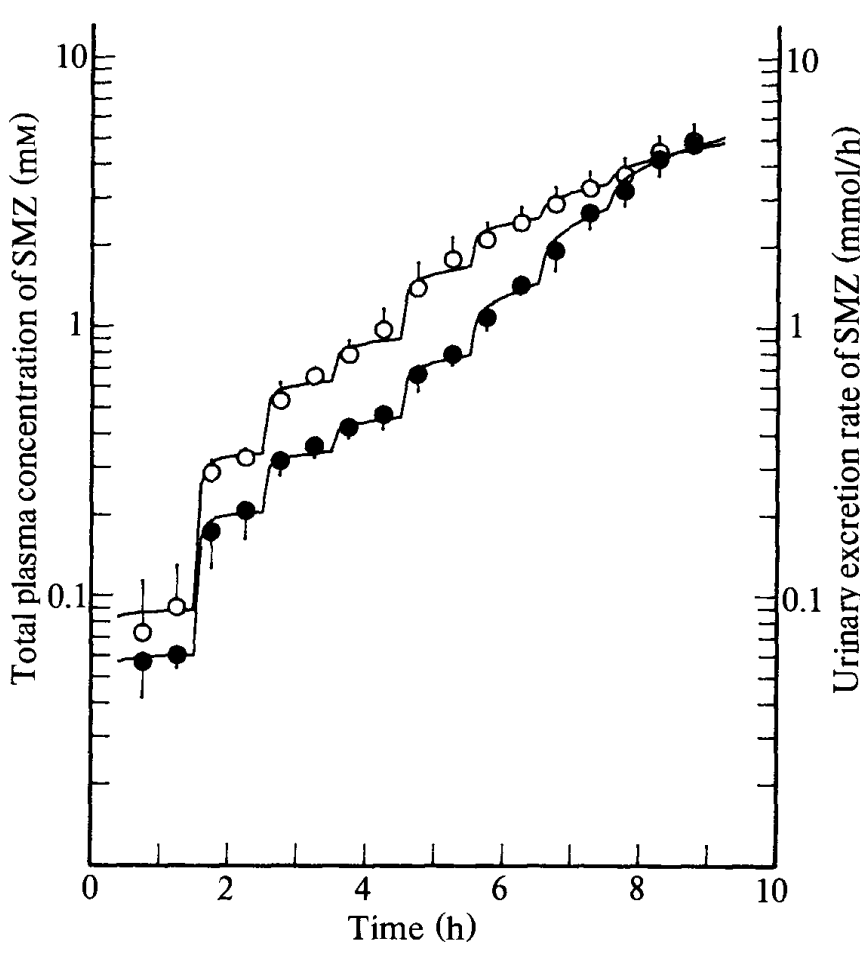

Fig. 8. Time Course of Plasma Concentration and Urinary Excretion Rate of SMZ during Changing the Infusion Rate in Steps

The plotted points represent the observed data (open circle, urinary excretion rate; closed circle, total plasma concentration) and the solid lines represent calculated values. Each experimental point is shown as the mean \pm S.D. $(n=5)$.

centrations was $1.30 \pm 0.03$ ( \pm S.D., $n=40)$ in the unbound plasma concentration range 0.01 to $2.0 \mathrm{mM}$ (data not shown). However, the half-

TABle III. Estimated Parameter Values of IOD and SMZ Disposition $^{a)}$

\begin{tabular}{llcc}
\hline & & IOD & SMZ \\
\hline$Q_{\mathrm{p}}$ & $(1 / \mathrm{h})$ & $4.02 \pm 0.23$ & $4.00 \pm 0.42$ \\
$\mathrm{GFR}$ & $(1 / \mathrm{h})$ & $\left.1.0^{b}\right)$ & $1.0^{b}$ \\
$V_{\mathrm{m}}$ & $(\mathrm{mmol} / \mathrm{h})$ & $3.01 \pm 0.16$ & $2.72 \pm 0.52$ \\
$K_{\mathrm{m}}$ & $(\mu \mathrm{M})$ & $10.0 \pm 8.5$ & $32.6 \pm 4.4$ \\
$\mathrm{CL}_{\mathrm{re}}$ & $(1 / \mathrm{h})$ & 0.0 & $0.030 \pm 0.016$ \\
$\mathrm{UR}$ & $(1 / \mathrm{h})$ & $0.11^{b)}$ & $0.1{ }^{b)}$ \\
$V_{\text {met }} / K_{\text {met }}(1 / \mathrm{h})$ & $0.611 \pm 0.120$ & - \\
$V_{\text {met }}$ & $(\mathrm{mmol} / \mathrm{h})$ & - & $1.27 \pm 0.27$ \\
$K_{\text {met }}$ & $(\mathrm{mM})$ & - & $0.857 \pm 0.279$ \\
$V_{\mathrm{d}}$ & $(1)$ & $0.959 \pm 0.168$ & - \\
\hline
\end{tabular}

a) Values are presented as mean \pm S.D. $b$ ) These values are experimentally measured. 
life of the dissociation of SMZ from erythrocytes $(31 \pm 4 \mathrm{~s})$ was sufficiently longer than the mean residence time of about $3 \mathrm{~s}$ (which was determined using Evans blue) of erythrocytes in the kidney. This indicated that there was little possibility of the contribution of SMZ being liberated from erythrocytes to the urinary excretion during the passage of the peritubular capillaries. Therefore, $Q_{\mathrm{p}}$ might agree with the effective renal plasma flow rather than the renal blood flow.

Figure 9 shows the urinary excretion rates of IOD and SMZ against the total plasma concentrations. As the plasma concentration of IOD increased, the urinary excretion rate initially increased almost linearly. This slope, i.e. the renal clearance of $3.74 \pm 0.32 \mathrm{1} / \mathrm{h}$ (at the total plasma levels from 0.05 to $0.8 \mathrm{mM}$ ) is an approximate of the renal plasma flow, though the unbound fraction in plasma varied from 0.37 to 0.65 (Fig. 4). The relationship between urinary excretion rates and plasma concentrations of SMZ did not give linear-ascending curves, but a concaveascending one, at low plasma concentrations. This indicated that the renal clearance of SMZ was limited by plasma protein binding. However, renal clearances referenced to unbound plasma concentration of SMZ and IOD at the total plasma concentration of $0.05 \mathrm{mM}$ were 38 and $9.51 / \mathrm{h}$, respectively. These values were much greater than the effective renal plasma flow. These facts indicated that the intrinsic

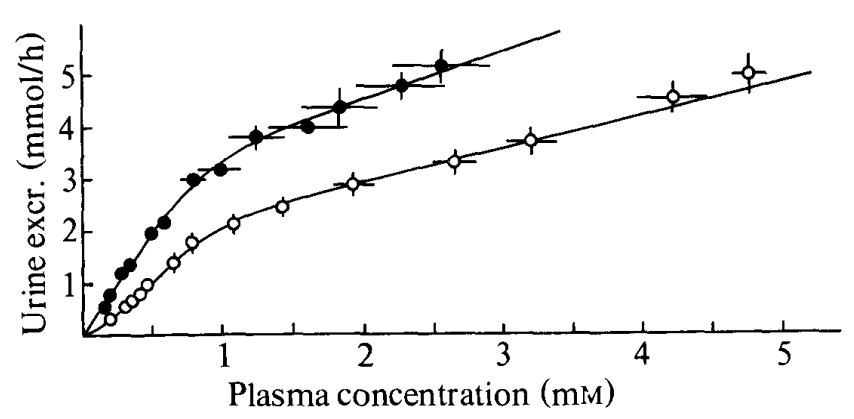

Fig. 9. Relationship between Urinary Excretion Rates and Total Plasma Concentrations of IOD and SMZ

The plotted points represent the observed data with plasma concentrations at $0.15 \mathrm{~mm}$ or above among the data shown in Figs. 7 and 8 . The solid lines represent the simulation values. Each point is the mean \pm S.E.M. (open circle, SMZ; closed circle, IOD). clearances $\left(V_{\mathrm{m}} / K_{\mathrm{m}}\right)$ were high and that unbound drug was liberated from plasma protein at the sites of tubular secretion. These suggested that the rates of secretion were fully (for IOD) or partially (for SMZ) limited by the renal plasma flow.

The differences in concentration between the renal artery and the renal vein decrease as drug concentrations at the site of secretion approach or exceed the dissociation constants for tubular secretion. Thus, a linear relationship between concentrations at the systemic circulation and at the sites of tubular secretion could not be always presumed, as described for IOD in the dog. ${ }^{4)} \mathrm{Al}$ though the importance of drug concentration at the sites of tubular secretion for a drug with a high extraction ratio has been emphasized, a similar finding appears to be valid for even a drug with a intermediate extraction ratio like SMZ. The fact that the parameter estimates for dissociation constants in the secretory process, i.e., for IOD and SMZ, 10 and $33 \mu \mathrm{M}$, respectively, agreed will with the corresponding dissociation constants of in vitro specific binding to renal cortez slices (Table I) strengthens the utility of the kinetic model based on drug concentrations at the sites of transport.

The quantitative interpretation of renal handling for drugs incorporated with glomerular filtration, secretion and reabsorption might be difficult and only a few models ${ }^{2,3,24)}$ have been presented assuming that secretion took place in the proximal region of the tubules and a constant fraction of the drug which was filtered and/or secreted would be subject to reabsorption form the distal region of the renal tubules. All the reabsorbed species were delivered to the renal vein.

In the present model, however, the reabsorbed species may be re-utilized as a source of tubular secretion, as designated by the terms of $\mathrm{CL}_{\text {re }} f_{\mathrm{p} \text {,out }} C_{\mathrm{p} \text {,out }}$ in Eq. 4 , Kidney is basically composed of the morphologic unit, i.e., the nephron and the surrounding vascular supply. These nephrons and vascular system are not only arranged in parallel but also in a hairpin loop shape. Furthermore the efferent arteriole in the cortical nephrons breaks up into a rich convoluted capillary network with anastomoses. 
Even in the juxtamedullary nephrons, the efferent arteriole gives up capillary nets enveloping the convolution of proximal and distal tubules. Therefore, the species reabsorbed through the peritubular interstitial fluids from the tubule may be not only delivered to the renal vein but also returned to the basolateral membrane in the proximal tubule and be re-utilized as a source of tubular secretion. In these circumstances, the vascular system can be considered as a well-stirred compartment as described by the present model. Although there is not ample evidence for the perfect mixing of the vascular system at present, one must always consider the possibility of the recirculation of reabsorbed species to elucidate the renal handling of drugs when tubular secretion and reabsorption both are operative.

In conclusion, the plasma concentration and urinary excretion time courses of IOD and SMZ were described in terms of the perfusion limited model characterizing the renal excretory processes. Estimated plasma flows and dissociation constants for tubular secretion were in good agreement with the effective renal plasma flow in rabbits and dissociation constants in renal slices accumulation, respectively. These facts indicated that the pharmacokinetic model based on physiological concepts was useful for the understanding of the drug elimination processes. The permeability constant of reabsorption $\left(\mathrm{CL}_{\mathrm{re}}\right)$ for SMZ was estimated from the concentration (ionized + unionized forms) difference between the lumen and the unbound plasma. However, passive reabsorption is inherently dependent on the concentrations of unionized species, and then can depend on urinary pH. Tubular reabsorption is also urine flow dependent. Further investigation is required in these respects.

\section{Appendix}

\section{The Estimation of Urinary Excretion Rates of IOD and SMZ}

When the total plasma concentration of IOD or SMZ in the artery $\left(C_{\mathrm{p}, \text { in }}\right)$ are given, the unbound plasma concentration in the artery $\left(C_{\mathrm{f}, \text { in }}\right)$ can be numerically calculated by the Newton-
Raphson iterative procedure using Eq. 12, in which the concentration of a displacer is zero.

When the unbound plasma concentration of IOD or SMZ in the vein $\left(C_{\mathrm{f}, \text { out }}\right)$ are given, the total plasma concentration in the vein $\left(C_{\mathrm{p}, \text { out }}\right)$ can be directly calculated using Eq. 12, in which each number of binding sites in the vein $\left(N_{1}(\mathrm{p})_{\text {out }}\right.$ and/or $\left.N_{2}(\mathrm{p})_{\text {out }}\right)$ is the product of $Q_{\mathrm{p}} /$ $\left(Q_{\mathrm{p}}\right.$-UR) and the number of binding sites in the systemic circulation. Therefore, in order to calculate the urinary excretion rate in Eq. 4 and/or the renal clearance in Eq. 7, the unbound plasma concentration of IOD or SMZ in the vein must be estimated from the total and unbound plasma concentrations in the artery. Substituting Eqs. 2 and 12 into Eq. 4 obtains the following equation of one unknown $\left(C_{\mathrm{f} \text {,out }}\right)$,

$f\left(C_{\mathrm{f}, \text { out }}\right)=0$

where

$$
\begin{aligned}
& f\left(C_{\mathrm{f}, \text { out }}\right)=Q_{\mathrm{p}} C_{\mathrm{p}, \text { in }}-\left(Q_{\mathrm{p}}-\mathrm{UR}\right) C_{\mathrm{f}, \text { out }}\left(1+\frac{N_{1}(\mathrm{p})_{\text {out }}}{K_{1}+C_{\mathrm{r}, \text { out }}}+\frac{N_{2}(\mathrm{p})_{\text {out }}}{K_{2}+C_{\mathrm{f}, \text { out }}}\right) \\
& -\left(\mathrm{GFR} C_{\mathrm{f}, \mathrm{in}}+\frac{V_{\mathrm{m}} C_{\mathrm{f}, \text { out }}}{K_{\mathrm{m}}+C_{\mathrm{f}, \text { out }}}+\mathrm{CL}_{\mathrm{re}} C_{\mathrm{f}, \text { out }}\right)\left(1-\frac{\mathrm{CL}_{\mathrm{re}}}{\mathrm{UR}+\mathrm{CL}_{\mathrm{re}}}\right)
\end{aligned}
$$

The estimation of $C_{\mathrm{f} \text {,out }}$ was achieved numerically using a Newton-Raphson iterative method.

\section{References}

1) K. Koshi, Y. Shirai, K. Katayama, M. Kakemi, M. Ueda, and T. Koizumi: A note on saturable and inhibitory kinetics of $p$-aminohippurate renal transport in rabbits, J. Pharmacobio-Dyn., 6, 161-169 (1983).

2) R. Hori, K. Sunayashiki, and A. Kamiya: Pharmacokinetic analysis of renal handling of sulfamethizole, $J$. Pharm. Sci., 65, 463-465 (1976).

3) G. Levy: Effect of plasma protein binding on renal clearance of drugs, J. Pharm. Sci., 69, 482-483 (1980).

4) P. Hekman and C. A. M. van Ginneken: Kinetic modeling of the renal excretion of iodopyracet in the dog, J. Pharmacokinet. Biopharm., 10, 77-92 (1982).

5) A. Tsuji, T. Yoshikawa, K. Nishide, H. Minami, M. Kimura, E. Nakashima, T. Terasaki, E. Miyamoto, C. H. Nightingale, and T. Yamana: Physiologically based pharmacokinetic model for $\beta$-lactam antibiotics I: Tissue distribution and elimination in rats, J. Pharm. Sci., 72, 1239-1251 (1983).

6) F. G. M. Russel, A. C. Wouterse, and C. A. M. van Ginneken: Physiologically based pharmacokinetic 
model for the renal clearance of phenolsulfonphthalein and the interaction with probenecid and salicyluric acid in the dog, J. Pharmacokinet. Biopharm., 15, 349-368 (1987).

7) N. Itoh, Y. Sawada, Y. Sugiyama, T. Iga, and M. Hanano: Kinetic analysis of rat renal tubular transport based on multiple-indicator dilution method, Am. J. Physiol., 251, F103-F114 (1986).

8) M. Rowland, L. Z. Benet, and G. G. Graham: Clearance concepts in pharmacokinetics, J. Pharmacokinet. Biopharm., 1, 123-136 (1973).

9) G. R. Wilkinson and D. G. Shand: A physiological approach to hepatic drug clearance, Clin. Pharmacol. Ther., 18, 377-390 (1975).

10) K. S. Pang and M. Rowland: Hepatic clearance of drugs. I. Theoretical considerations of a "well-stirred" model and a "parallel tube" model. Influence of hepatic blood flow, plasma and blood cell binding, and the hepatocellular enzymatic activity on hepatic drug clearance, J. Pharmacokinet. Biopharm., 5, 625-653 (1977).

11) D. D. S. Tang-Liu, R. L. Williams, and S. Riegelman: Dependence of renal clearance on urine flow: A mathematical model and its application, J. Pharm. Sci., 72, 154-158 (1983).

12) S. Hall and M. Rowland: Relationship between renal clearance, protein binding and urine flow for digitoxin, a compound of low clearance in the isolated perfused rat kidney, J. Pharmacol. Exp. Ther., 227, 174-179 (1983).

13) I. Komiya: Urine flow dependence of renal clearance and interrelation of renal reabsorption and physicochemical properties of drugs, Drug Metab. Disp., 14, 239-245 (1986).

14) R. Hori, K. Sunayashiki, and A. Kamiya: Tissue distribution and metabolism of drugs. I. Quantitative investigation on renal handling of phenolsulfonphthalein and sulfonamides in rabbits, Chem. Pharm. Bull., 26,
$740-745$ (1978).

15) K. Katayama, H. Ohtani, A. Murai, M. Kakemi, and T. Koizumi: Kinetic studies on drug disposition in rabbits. II. Dose dependent pharmacokinetics of sulfamethizole, J. Pharmacobio-Dyn., 13, 108-119 (1990).

16) P. Hekman and C. A. M. van Ginneken: Rapid determination of renal contrast media in biological fluids by means of high-performance liquid chromatography, J. Chromatogr., 182, 492-495 (1980).

17) E. Wahlin-Boll and A. Melander: High-performance liquid chromatographic determination of glipizide and some other sulfonylurea drugs in serum, J. Chromatogr., 164, 541-546 (1979).

18) T. Koizumi, M. Ueda, S. Takada, and K. Tada: Kinetics of $p$-aminohippurate renal excretion in rabbits, $J$. Pharmacobio-Dyn., 1, 39-48 (1978).

19) Z. Dische and E. Borenfreund: A new spectrophotometric method for the detection and determination of keto sugars and trioses, J. Biol. Chem., 192, 583-587 (1951).

20) M. Berman, E. Shahn, and M.F. Weiss: The routine fitting of kinetic data to models: A mathematical formalism for digital computers, Biophys. J., 2, 275-287 (1962).

21) K. Katayama, H. Ohtani, H. Mizuno, T. Taniguchi, M. Kakemi, and T. Koizumi: Kinetic studies on drug disposition in rabbits. III. Effect of tolbutamide on renal excretion of sulfamethizole, J. Pharmacobio-Dyn., 13, 120-129 (1990).

22) K. Katayama, E. Tsubota, M. Endoh, M. Kakami, and T. Koizumi: Effect of iodopyracet on renal excretion of sulfamethizole in rabbits, J. Pharmacobio-Dyn., in press.

23) F. Kill: Dynamics of renal proximal tubular secretion, Nature (London), 189, 927-928 (1961).

24) E. R. Garrett: Pharmacokinetics and clearances related to renal processes, Int. J. Clin. Pharmacol., 16, 155-172 (1978). 